\title{
ON SIMPLE REDUCIBLE DEPTH-TWO LIE ALGEBRAS WITH CLASSICAL REDUCTIVE NULL COMPONENT
}

\author{
THOMAS B. GREGORY
}

\begin{abstract}
We classify the simple finite-dimensional reducible graded Lie algebras of the form $L_{-2} \oplus L_{-1} \oplus L_{0} \oplus L_{1} \oplus \cdots \oplus L_{k}$ over an algebraically closed field of characteristic greater than 3 , where $L_{0}$ is reductive and classical such that no nonzero element of the center of $L_{0}$ annihilates $L_{-2}$ and where $L_{-1}$ is the sum of two proper $L_{0}$-submodules.
\end{abstract}

In [2], the present author showed that if the null component of a simple finitedimensional Lie algebra of the form

$$
L=L_{-2} \oplus L_{-1} \oplus L_{0} \oplus L_{1} \oplus \cdots \oplus L_{k}
$$

contains no nonzero abelian ideal which annihilates $L_{-2}$, and if $L_{-1}=S+T$, where $S$ and $T$ are proper $L_{0}$-submodules of $L_{-1}$, then $S$ and $T$ are abelian irreducible $L_{0}$-submodules, and $L_{-1}=S \oplus T$. In addition, $L$ possesses an irreducible transitive gradation of the form

$$
L=M_{-1} \oplus M_{0} \oplus \cdots \oplus M_{[k / 2]},
$$

such that (by interchanging the names of $S$ and $T$ if necessary) $M_{0}=T \oplus L_{0} \oplus$ $\left[L_{2}, S\right]$ and $\left[L_{2}, S\right]=\{0\}$ if $k$ is odd.

In this paper, we strengthen the hypotheses on $L_{0}$; we assume that $L_{0}$ is classical and reductive and that $A \mathrm{An}_{L_{0}} L_{-2}$ contains no nonzero element of the center of $L_{0}$. We then prove that $L$ is either classical or of Cartan type. Specifically, we prove the following

THEOREM. Let $L$ be a simple finite-dimensional reducible graded Lie algebra of the form (1) over an algebraically closed field of characteristic greater than 3, and suppose that $L_{0}$ is a classical reductive Lie algebra no nonzero element of whose center annihilates $L_{-2}$, and that $L_{-1}=S+T$, where $S$ and $T$ are proper $L_{0}$-submodules of $L_{-1}$. Then $L$ is classical or of Cartan type.

(For definitions of the terms "transitive" and "irreducible" as they are used in this paper, see [1].)

The proof of this theorem will be carried out by means of a series of lemmas. We begin by recalling certain of the lemmas of [2] for which we will have use here. The numbers in parentheses after the first 10 lemma numbers are those by which the lemmas were designated in [2]. Proofs of these lemmas can be found in [2] (or [1]). In the first 4 lemmas, we assume only that $L$ is a simple graded Lie algebra of the form (1).

Received by the editors April 13, 1981.

1980 Mathematics Subject Classification. Primary 17B20.

(C) 1982 American Mathematical Society 0002-9939/81/0000-1064/\$02.25 
LEMMA 1(1). $L_{-2}$ is an irreducible $L_{0}$-module. Furthermore, $\left[L_{-1}, L_{-1}\right] \neq$ $\{0\}$, so $\left[L_{-1}, L_{-1}\right]=L_{-2} ;$ also, $L_{-1}=\left[L_{-2}, L_{1}\right]$. Lastly, $\left[L_{-1}, x\right] \neq\{0\}$ for all $x \notin L_{-2}$.

LEMMA 2(2). $L_{k}$ is an irreducible $L_{0}$-module, and $L_{j-1}=\left[L_{j}, L_{-1}\right]$ for all $j$, $-1 \leq j \leq k$.

LEMMA 3(3). We have that $\left[L_{0}, L_{k}\right] \neq\{0\}$ and that $\left[L_{-2}, L_{i}\right] \neq\{0\}$ for all $i$, $0 \leq i \leq k$.

LEMMA 4(4). $L_{-1}$ contains no proper nonabelian $L_{0}$-submodule.

LEMMA $5(6) . \quad\left[S,\left[S, L_{2}\right]\right]=\{0\}$.

LEMMA 6(9). $\operatorname{Ann}_{L_{i}} L_{-2}=\{0\}$ for all $i, 1 \leq i \leq k$.

LEMMA $7(10) . \quad L_{-1}=S \oplus T$, where $S$ and $T$ are irreducible abelian $L_{0}$ submodules of $L_{-1}$.

LEMMA 8(13). $L_{2 i-1}=\left[L_{2 i}, S\right] \oplus\left[L_{2 i}, T\right]$ for all $i, 0 \leq i \leq[k / 2]$.

LEMMA 9(14). $\operatorname{Ann}_{L_{2 i-1}} S=\left[L_{2 i}, S\right]$ for all $i, 0 \leq i \leq[k / 2]$.

LEMMA $10(15)$. $\left[\left[L_{2 i}, S\right],\left[L_{2 j}, S\right]\right]=\{0\}$ for all $i$ and $j, 0 \leq i, j \leq[k / 2]$.

LEMMA 11. $\left[L_{0}, S\right] \neq\{0\}$.

Proof. By Lemma 8, we have that $L_{-1}=\left[L_{0}, S\right]+\left[L_{0}, T\right]$. If $\left[L_{0}, S\right]=\{0\}$, we would have by Lemma 4 that $\left[L_{-1}, L_{-1}\right] \subseteq[T, T]=\{0\}$, contrary to Lemma 1. Q.E.D.

LEMMA 12. $\operatorname{Ann}_{\left[L_{2}, S\right]} T=\{0\}$.

Proof. By Lemma 9, $\operatorname{Ann}_{\left[L_{2}, S\right]} T \subseteq \operatorname{Ann}_{L_{1}} T=\left[L_{2}, T\right]$. By Lemma 8, then, we have that $\operatorname{Ann}_{\left[L_{2}, S\right]} T \subseteq\left[L_{2}, T\right] \cap\left[L_{2}, S\right]=\{0\}$. Q.E.D.

LEMMA 13. $\left[L_{2}, S\right] \neq\{0\}$.

Proof. If $\left[L_{2}, S\right]=\{0\}$, then by Lemma $8, L_{1}=\left[L_{2}, T\right]$. Hence, we have by Lemma 1 that $L_{-1}=\left[L_{-2}, L_{1}\right]=\left[L_{-2},\left[L_{2}, T\right]\right]=\left[\left[L_{-2}, L_{2}\right], T\right] \subseteq T$, so that $\{0\} \neq\left[L_{-1}, L_{-1}\right] \subseteq[T, T]$, contrary to Lemma 7. Q.E.D.

LEMMA 14. $\operatorname{Ann}_{T}\left[L_{2}, S\right]=\{0\}$.

Proof. Since $T$ is an irreducible $L_{0}$-module by Lemma 7 , it follows that if $\operatorname{Ann}_{T}\left[L_{2}, S\right] \neq\{0\}$, then $\operatorname{Ann}_{T}\left[L_{2}, S\right]=T$. Hence, we would have by Lemma 5 that $\left[L_{-1},\left[L_{2}, S\right]\right]=\left[S \oplus T,\left[L_{2}, S\right]\right] \subseteq\left[S,\left[L_{2}, S\right]\right]+\left[T,\left[L_{2}, S\right]\right]=\{0\}$. Then Lemma 1 yields that $\left[L_{2}, S\right]=\{0\}$, contrary to Lemma 13 . Q.E.D.

LEMMA 15. There exist elements $c$ and $q$ of the center of $\left[\left[L_{2}, S\right], T\right]$ and the center of $\left[\left[L_{2}, T\right], S\right]$, respectively, such that $\operatorname{Ann}_{\left[\left[L_{2}, S\right], T\right]}\left[L_{2}, S\right]=\operatorname{Ann}_{\left[\left[L_{2}, S\right], T\right]} T \subseteq\langle c\rangle$ and $\operatorname{Ann}_{\left[\left[L_{2}, T\right], S\right]}\left[L_{2}, T\right]=\operatorname{Ann}_{\left[\left[L_{2}, T\right], S\right]} S \subseteq\langle q\rangle$. 
Proof. Set $B=\operatorname{Ann}_{\left[\left[L_{2}, S\right], T\right]}\left[L_{2}, S\right] . \quad$ If $[B, T] \neq\{0\}$, then by Lemma $7,[B, T]=T$, so $\left[\left[L_{2}, S\right], T\right]=\left[\left[L_{2}, S\right],[B, T]\right]=\left[B,\left[\left[L_{2}, S\right], T\right]\right] \subseteq B$; hence, $\left[\left[L_{2}, S\right],\left[\left[L_{2}, S\right], T\right]\right]=\{0\}$. Let $y$ be any element of $\left[L_{2}, S\right]$. Then $[T, y]$ is an $\left[\left[L_{2}, S\right], T\right]$-submodule (i.e., an ideal) of $\left[\left[L_{2}, S\right], T\right]$. Because $y$ is fixed, each element of $[T, y]$ is of the form $[t, y]$, where $t \in T$. Thus, let $t$ be any element of $T$. We have by Lemma 7 that $\{0\}=(\operatorname{ad} y)^{2}(\operatorname{ad} t)^{2}[T, y]=2(\operatorname{ad}[y, t])^{2}[T, y]$. By Engel's Theorem, then, $[T, y]$ is nilpotent. But $[T, y]$ is an ideal of $\left[\left[L_{2}, S\right], T\right]$, so $[T, y]$ is an ideal of the reductive $L_{0}$, and, hence, must be in the center of $L_{0}$, as must all of $\left[\left[L_{2}, S\right], T\right]$, because $y$ was an arbitrary element of $\left[L_{2}, S\right]$. Consequently, we have by Schur's Lemma that each element $x$ of $\left[\left[L_{2}, S\right], T\right]$ acts as a scalar $\xi(x)$ on $T$, which is irreducible by Lemma 7 . As before, let $t$ be any element of $T$. By Lemma 14 , there exists a $z \in\left[L_{2}, S\right]$ such that $[t, z] \neq 0$. For any $x \in\left[\left[L_{2}, S\right], T\right]$, we have $0=[x,[t, z]]=[[x, t], z]=\xi(x)[t, z]$. Thus, $\xi(x)=0$ for all $x \in\left[\left[L_{2}, S\right], T\right]$, so that $\{0\}=\left[\left[\left[L_{2}, S\right], T\right], T\right]=[B, T]$.

Now consider $C=\operatorname{Ann}_{\left[\left[L_{2}, S\right], T\right]} T$. If $\left[C,\left[L_{2}, S\right]\right] \neq\{0\}$, then $\left[T,\left[C,\left[L_{2}, S\right]\right]\right] \neq$ $\{0\}$, since $\operatorname{Ann}_{\left[L_{2}, S\right]} T=\{0\}$ by Lemma 12. Hence, we have $\{0\} \neq\left[T,\left[C,\left[L_{2}, S\right]\right]\right]$ $=\left[C,\left[T,\left[L_{2}, S\right]\right]\right]=[C, C]$, the semisimple part of $C$, so that $\left[T,\left[T,\left[C,\left[L_{2}, S\right]\right]\right]\right]=$ $\{0\}$. Arguing as before, we have by Lemma 10 that if $x \in T$ and $y \in\left[C,\left[L_{2}, S\right]\right]$ then $\{0\}=(\operatorname{ad} x)^{2}(\operatorname{ad} y)^{2}\left[T,\left[C,\left[L_{2}, S\right]\right]\right]=2(\operatorname{ad}[x, y])^{2}\left[T,\left[C,\left[L_{2}, S\right]\right]\right]$ so that $\left[T,\left[C,\left[L_{2}, S\right]\right]\right]$ is central in $\left[\left[L_{2}, S\right], T\right]$, contrary to our conclusion above that it must be nonzero and semisimple. Thus, we must have that $\left[C,\left[L_{2}, S\right]\right]=\{0\}$, so that $C=B$.

Suppose that $B$ is nonzero, and let $c \in B \backslash\{0\}$. By Lemmas 1 and $7,\{0\} \neq$ $\left[c, L_{-1}\right]=[c, S]$. Since $c$ must be in the center of the reductive $L_{0}$, and since $S$ is an irreducible $L_{0}$-module by Lemma 7 , we have by Schur's Lemma that for all $s \in S,[s, c]=\gamma s$ for some nonzero scalar $\gamma$. If $d$ is any other nonzero element of $B$, then of course $[s, d]=\delta s, \delta \neq 0$, for all $s \in S$. Then if $v=s+t(s \in$ $S, t \in T)$ is any element of $L_{-1}$, we have that $[v, c / \gamma-d / \delta]=s-s=0$, so by Lemma $1, c / \gamma-d / \delta=\{0\}$, and $B=\langle c\rangle$. The rest of the lemma follows by symmetry. Q.E.D.

LEMMA 16. If no nonzero element of the center of $L_{0}$ annihilates $L_{-2}$, then either $c=0$ or $q=0$, where $c$ and $q$ are as in Lemma 15.

Proof. If both $c$ and $q$ are nonzero, then there exist nonzero scalars $\alpha$ and $\beta$ such that $[w, c]=\alpha w$ and $[w, q]=\beta w$ for all $w \in L_{-2}$, which is an irreducible $L_{0}$-module by Lemma 1 . Then $\left[L_{-2}, c / \alpha-q / \beta\right]=\{0\}$, so $c / \alpha=q / \beta$; hence $c$ annihilates both $S$ and $T$, and we have that $\left[L_{-1}, c\right]=\{0\}$, contrary to Lemma 1. Q.E.D.

LEMMA 17. Suppose no nonzero element of the center of $L_{0}$ annihilates $L_{-2}$. Let $C$ be as in the proof of Lemma 15. If $k$ is even, we can assume that $C=\{0\}$. If $k$ is odd, we have that $L_{0}=\left[\left[L_{2}, S\right], T\right]$.

Proof. If $k$ is even, then $S$ and $T$ are symmetric, so by relabelling if necessary, we can assume by Lemmas 15 and 16 that $C=B=\{0\}$.

If $k$ is odd, then $\left[S, L_{k}\right]=\{0\}$ (see (2)ff). Then by Lemmas 6,7 , 8, and 9 $L_{k}\left(\operatorname{ad} L_{-2}\right)^{(k+1) / 2}=S$, so $\left[\left[L_{2}, T\right], S\right] \subseteq\left[L_{k},\left[\left[L_{2}, T\right], L_{-2}\right]\right]\left(\operatorname{ad} L_{-2}\right)^{(k-1) / 2}=$ $\left[L_{k}, T\right]\left(\operatorname{ad} L_{-2}\right)^{(k-1) / 2}=\left[L_{k}\left(\operatorname{ad} L_{-2}\right)^{(k-1) / 2}, T\right] \subseteq\left[\left[L_{2}, S\right], T\right]$. Thus, by Lemmas 
2,7 , and 5 ,

$$
L_{0}=\left[\left[L_{2}, L_{-1}\right], L_{-1}\right]=\left[\left[L_{2}, S\right], T\right]+\left[\left[L_{2}, T\right], S\right] \subseteq\left[\left[L_{2}, S\right], T\right],
$$

so that $L_{0}=\left[\left[L_{2}, S\right], T\right]$. Q.E.D.

Set $N=T \oplus\left[\left[L_{2}, S\right], T\right] \oplus\left[L_{2}, S\right]$. Then $N$ is a graded Lie algebra, where for all $i,-1 \leq i \leq 1$, the $i$ th gradation space of $N$ is $N \cap L_{i}$.

LEMMA 18. $N=T \bigoplus\left[\left[L_{2}, S\right], T\right] \oplus\left[L_{2}, S\right]$ is an irreducible graded Lie algebra whenever $L_{0}$ contains no nonzero central element which annihilates $L_{-2}$.

Proof. Let $Q$ be a nonzero $\left[\left[L_{2}, S\right], T\right]$-submodule of $T$. Then $\left[\left[L_{2}, S\right], Q\right]$ is an $\left[\left[L_{2}, S\right], T\right]$-submodule of $\left[\left[L_{2}, S\right], T\right]$. Furthermore, since $L_{0}$ is reductive, $L_{0}=\left[\left[L_{2}, S\right], T\right] \oplus R$ for some ideal $R$ of $L_{0}$ such that $\left[R,\left[\left[L_{2}, S\right], T\right]\right]=\{0\}$. Hence, $\left[\left[L_{2}, S\right], Q\right]$ is an $L_{0}$-submodule of $\left[\left[L_{2}, S\right], T\right]$, so $\left[\left[\left[L_{2}, S\right], Q\right], T\right]$ is an $L_{0^{-}}$ submodule of $T$. By Lemma 7, [[[L $\left.\left.\left.L_{2}, S\right], Q\right], T\right]$ cannot be a proper submodule of $T$. If $\{0\}=\left[\left[\left[L_{2}, S\right], Q\right], T\right]=\left[\left[\left[L_{2}, S\right], T\right], Q\right]$, then $Q \subseteq \operatorname{Ann}_{T}\left[\left[L_{2}, S\right], T\right]=T$, since $\operatorname{Ann}_{T}\left[\left[L_{2}, S\right], T\right]$ is an $L_{0}$-submodule of the irreducible $L_{0}$-submodule $T$. Let $C$ be as above in the proof of Lemma 15 . We have that $\left[\left[L_{2}, S\right], T\right] \subseteq C$.

If $k$ is odd, we have by Lemma 17 that $L_{0}=\left[\left[L_{2}, S\right], T\right] \subseteq \bar{C}$, so that $\left[L_{0}, T\right]=$ $\{0\}$, contrary to Lemma 11 . If $k$ is even, we have by Lemma 17 that $C=\{0\}$. But then we would have, for example, that $\operatorname{Ann}_{\left[L_{2}, S\right]} T=\left[L_{2}, S\right] \neq\{0\}$ by Lemma 13 , contrary to Lemma 12 . Hence, we must have that $\left[\left[\left[L_{2}, S\right], Q\right], T\right]=T$, and we have

$$
Q \supseteq\left[\left[\left[L_{2}, S\right], T\right], Q\right]=\left[\left[\left[L_{2}, S\right], Q\right], T\right]=T . \quad \text { Q.E.D. }
$$

LEMMA 19. For all $i,-2 \leq i \leq k-1, L_{i}$ is spanned by weight vectors of the form $[x, y]$, where $x$ is a weight vector of $L_{-1}$ and $y$ is a weight vector of $L_{i+1} . L_{k}$ is spanned by weight vectors, also.

ProOF. $S, T$, and $L_{k}$ are irreducible $L_{0}$-modules by Lemmas 2 and 7 . Hence, each is equal to the set of all weight vectors which it contains, since each of these sets is an $L_{0}$-submodule which is nonempty due to the fact that each of the $L_{0}$-modules $S, T$, and $L_{k}$ is a finite-dimensional vector space over an algebraically closed field, and the Cartan subalgebra of $L_{0}$ is commutative. Lemma 19 now follows from Lemma 2. Q.E.D.

PROOF OF THEOREM. By Lemma 3, and our assumption that the center of $L_{0}$ contains no nonzero element which annihilates $L_{-2}$, we can apply the Theorem of [2] to show that $L$ possesses a gradation of the form (2), where $M_{0}=T \oplus L_{0} \oplus$ $\left[L_{2}, S\right]$. If $c$ is as in Lemma 15 then by Lemmas 12, 14, and 16, the Lie algebra $N /\langle c\rangle$ is a transitive graded Lie algebra. By Lemma $18, N$ is an irreducible graded Lie algebra. In view of Lemmas 12 and 19 we can use the arguments of Lemma 8 and $\S 4$ of $[3]$ to show that the representation of $\left[\left[L_{2}, S\right], T\right] /\langle c\rangle$ in $T$ is restricted. Then the hypotheses of Theorem 3 of [4] are satisfied, so $N /\langle c\rangle$ must be classical and reductive. Thus, if $k$ is even, we have from Lemma 17 that $M_{0}=N+L_{0}$ is classical and reductive. If $k$ is odd, then by Lemma 17 again, $M_{0}=N+L_{0} \subseteq N$ modulo its center (which by Lemmas 15 and 16 is of dimension at most one) is simple. In view of (for example) the paragraph following Theorem 3 of [4], we can now use the proof of the Theorem of [3] to conclude that $L$ is classical or of Cartan type. 


\section{REFERENCES}

1. T. B. Gregory, A characterization of the contact Lie algebras, Proc. Amer. Math. Soc. 82 (1981), 505-511.

2. __ On simple reducible Lie algebras of depth two, Proc. Amer. Math. Soc. 83 (1981), 31-35.

3. __ Simple Lie algebras with classical reductive null component, J. Algebra 63 (1980), 484-493.

4. V. G. Kac, The classification of the simple Lie algebras over a field with non-zero characteristic, Izv. Akad. Nauk SSSR Ser. Mat. 34 (1970), 385-408; English transl., Math. USSR Izv. 4 (1970), 391-413.

Department of Mathematics, The Ohio State University at Mansfield, MANSFIELD, OHIO 44906 